\title{
Haplotyping and copy number estimation of the highly polymorphic human beta-defensin locus on 8 p23 by 454 amplicon sequencing
}

\author{
Stefan Taudien*1, Marco Groth1', Klaus Huse1, Andreas Petzold1', Karol Szafranski1', Jochen Hampe3, Philip Rosenstiel2, \\ Stefan Schreiber ${ }^{2,3}$ and Matthias Platzer ${ }^{1}$
}

\begin{abstract}
Background: The beta-defensin gene cluster (DEFB) at chromosome 8p23.1 is one of the most copy number (CN) variable regions of the human genome. Whereas individual DEFB CNs have been suggested as independent genetic risk factors for several diseases (e.g. psoriasis and Crohn's disease), the role of multisite sequence variations (MSV) is less well understood and to date has only been reported for prostate cancer. Simultaneous assessment of MSVs and CNs can be achieved by PCR, cloning and Sanger sequencing, however, these methods are labour and cost intensive as well as prone to methodological bias introduced by bacterial cloning. Here, we demonstrate that amplicon sequencing of pooled individual PCR products by the 454 technology allows in-depth determination of MSV haplotypes and estimation of DEFB CNs in parallel.

Results: Six PCR products spread over $\sim 87 \mathrm{~kb}$ of DEFB and harbouring 24 known MSVs were amplified from 11 DNA samples, pooled and sequenced on a Roche 454 GS FLX sequencer. From $\sim 142,000$ reads, 120,000 haplotype calls $(\mathrm{HC})$ were inferred that identified 22 haplotypes ranging from 2 to 7 per amplicon. In addition to the 24 known MSVs, two additional sequence variations were detected. Minimal CNs were estimated from the ratio of HCs and compared to absolute CNs determined by alternative methods. Concordance in CNs was found for 7 samples, the CNs differed by one in 2 samples and the estimated minimal CN was half of the absolute in one sample. For 7 samples and 2 amplicons, the 454 haplotyping results were compared to those by cloning/Sanger sequencing. Intrinsic problems related to chimera formation during PCR and differences between haplotyping by 454 and cloning/Sanger sequencing are discussed.

Conclusion: Deep amplicon sequencing using the 454 technology yield thousands of HCs per amplicon for an affordable price and may represent an effective method for parallel haplotyping and CN estimation in small to medium-sized cohorts. The obtained haplotypes represent a valuable resource to facilitate further studies of the biomedical impact of highly $\mathrm{CN}$ variable loci such as the beta-defensin locus.
\end{abstract}

\section{Background}

Since the pioneering publication of Margulies et al. [1] many researchers have demonstrated the versatility of the massively parallel 454 pyrosequencing technology. The method has been successfully applied to a large diversity of targets such as nuclear and organellar genomes [2-4], transcriptomes [5-8], cloned DNA (e.g. BACs) [9], and PCR products (amplicons) [10]. This broad spectrum is

\footnotetext{
* Correspondence: stau@fli-leibniz.de

1 Leibniz Institute for Age Research - Fritz Lipmann Institute, D-07745 Jena, Germany

Full list of author information is available at the end of the article
}

complemented by a wide range of analyses, including epigenetic features [11,12], genome diversity $[13,14]$ and ancient DNA [15].

In principle, the 454 technology should also be applicable for haplotyping copy number $(\mathrm{CN})$ variable loci such as the beta-defensin gene cluster (DEFB) at human chromosome 8p23.1 (Fig. 1). This locus is intensively studied [16-26] is recognized as one of the most dynamic regions of the human genome [27] and was proven to be variable in $\mathrm{CN}$ as a whole [28]. Individual DEFB CNs and specific DEFB haplotypes have been shown to be associated with 


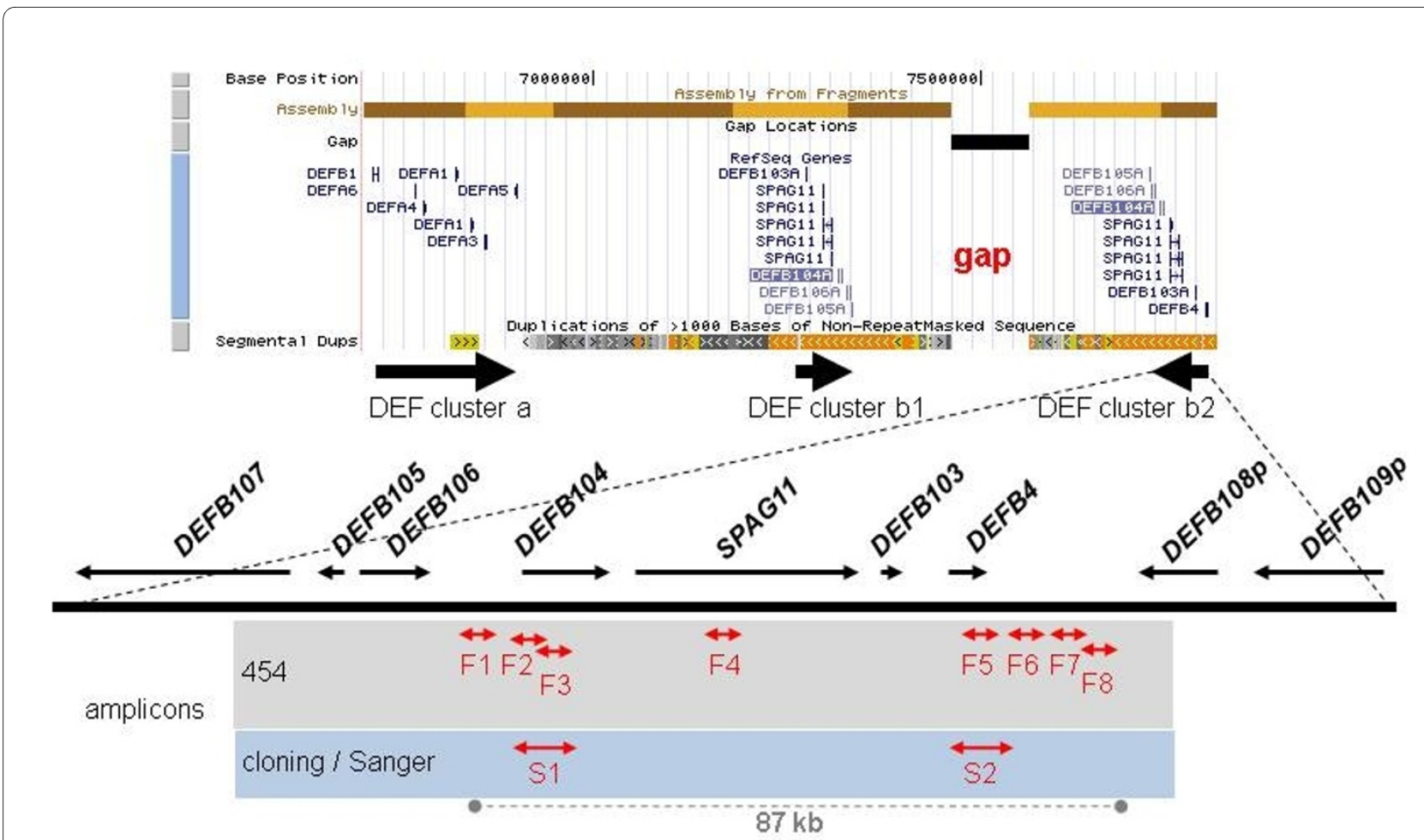

Figure 1 Human defensin gene clusters at 8p23.1 (NCBI build 36, hg18) and location of the amplicons analyzed by 454 (F1-8) and cloning/ Sanger (S1-2) sequencing within the proximal DEFB (DEF cluster b2).

psoriasis, Crohn's disease and prostate cancer $[18,20,29,30]$. Within the 2 DEFBs assembled in the human reference sequence (234 and $224 \mathrm{~kb}$; hg18, build 36.1, chr8:7,156,778-7,391,276 and chr8:7,669,629$7,893,454)$, a total of 2,971 single nucleotide polymorphisms (SNP) are annotated in dbSNP (build 130) which we address, more appropriately, as multisite variations (MSV) [31].

A potential method to assess individual haplotypes at such loci is the amplification of an MSV-containing region by PCR [16]. Subsequently, the amplicons are subcloned in plasmids, individual bacterial clones are Sanger sequenced and haplotypes are determined according to the bases at the polymorphic positions. This approach, however, has inherent problems. First, individual $\mathrm{CNs}$ can vary between 2 and 12 DEFB copies per diploid genome. Theoretically, each genotype can comprise a number of haplotypes equal to this $\mathrm{CN}$.

For individuals with many copies there is a high risk of missing rare haplotypes due to the decreased per-copy sequencing depth of 100-200 reads per genotype in a typical cloning/Sanger sequencing approach.

Second, PCR on complex templates may result in chimera formation by PCR-mediated recombination, creating artificial, false-positive haplotypes [32]. Third, the bacterial cloning step may introduce a systematic bias in the observed haplotype frequencies due to differences in the compatibility of clones with the host's lifecycle. To minimize the risk of spurious haplotyping by PCR/subcloning, independent PCR products have to be analyzed and large numbers of clones should be sequenced. This necessary quality control is usually too costly and labour intensive for high-throughput applications. In addition to assessing sequence variations, haplotyping has also been used for the measurement of DEFB CNs [16,17]. Absolute $\mathrm{CNs}$, however, cannot be determined and even reliable relative $\mathrm{CN}$ measurement by this method is challenging. E. g. in the simplest case of just 2 copies and 2 haplotypes in an MSV spanning region, relatively small numerical deviations from the theoretical ratio of 1.0 (1:1) may result in quite different minimal $\mathrm{CN}$ estimations, as 1.2 (6:5) indicates 11 copies, 1.25 (5:4) indicates 9 and 1.33 (4:3) indicates 7.

For effective and accurate determinations of absolute DEFB CN, multiplex ligation-dependent probe amplification (MLPA) [28,33], real-time PCR [26,34,35], multiplex amplifiable probe hybridisation combined with restriction enzyme digest variant ratios (MAPH/REDVR) [36] and different variants of paralog ratio tests (PRT) $[18,28,36]$ have been applied, however, they do not provide haplotype information. Knowledge, however, of both DEFB CN [20,29,30] and haplotypes [18] is of great 
importance with respect to clinically important phenotypes. Therefore, an ideal method for DEFB analysis and association studies would provide both CNs and haplotypes at once.

Application of massively parallel 454 technology to PCR amplicons allows extreme in-depth sequencing resulting from the huge amount of data generated by a single instrument run. Given a 250 bp amplicon and assuming 12,000 reads of $\sim 250 \mathrm{bp}$ length per $1 / 16^{\text {th }}$ segment of a GS FLX sequencer picotiterplate (GS FLX amplicon protocol), 16 PCR products can be sequenced in parallel with $12,000 \times$ coverage. This over-sequencing can be reasonably reduced using a multiplex approach, pooling either different amplicons generated from the same DNA $[37,38]$ or the same amplicons derived from multiple DNAs using a barcoding strategy [39]. However, in the example above, pooling of 8 amplicons should still lead to a mean coverage of 1,500. This amount of sequences per individual amplicon is still sufficient to reduce the probability for haplotyping errors as described above. Furthermore, 454 amplicon sequencing does not require bacterial cloning, avoiding the danger of respective biases. As result, it delivers a multiple of individual sequences for a given amplicon compared to a routine cloning/Sanger sequencing strategy.

We took advantage of this methodological approach for DEFB haplotyping and $\mathrm{CN}$ estimation by 454 sequencing to study the DEFB locus in 11 DNA samples from publicly available lymphoblastoid cell lines (LCL). The results are compared to those obtained by the cloning/Sanger approach, and to CN measurements using MLPA, MAPH/REDVR and PRT.

\section{Methods \\ DNA}

Genomic DNA from the CEPH Resources (NA12760), the DNA Polymorphism Discovery Resource Collection (NA15029, NA15213, and NA15385) and the [40] (NA18502, NA18552, NA18858, NA19140) were purchased at the Coriell cell depository http://ccr.coriell.org/ . DNAs C0140, C0766 and C0913 were obtained from the European Collection of Cell Cultures ECACC [41]. All 11 DNAs are derived from human LCLs, which were established from individuals of different ancestry, i.e. Caucasian (C0140, C0766, C0913, and NA12760), Yoruba (NA18502, NA18858, and NA19140) and Han Chinese (NA18552). For NA15029, NA15213, and NA15385 no information about the ethnicity of the donors is provided by the Discovery Resource Collection.

\section{4 amplicon sequencing}

PCR methods have been established to generate 8 DEFB specific amplicons (F1 to F8) using 454 adaptors A and $B$ containing fusion primers (HPLC purified, TIBMolBio; Additional file 1, Table S1). PCRs were carried out with
50 ng DNA using the "Advantage" mixed polymerase containing both $\mathrm{Taq}$ and a proofreading enzyme (Clontech 639201 ) and 35 cycles of $94^{\circ} \mathrm{C}$ for $30 \mathrm{~s}, 57^{\circ} \mathrm{C}$ for $1 \mathrm{~min}$ and $72^{\circ} \mathrm{C}$ for $2 \mathrm{~min}$ followed by $72^{\circ} \mathrm{C}$ for $10 \mathrm{~min}$. Concentrations of the PCR products were measured by Nanodrop and the Quant-IT ${ }^{\circ}$ PicoGreen ds DNA assay (Invitrogen). All amplicons derived from an individual DNA sample were mixed in an equimolar ratio $\left(\sim 4 \times 10^{10}\right.$ molecules per amplicon). The pools were diluted and subjected to emulsion PCR following the FLX emPCR protocol for amplicons (Roche Diagnostics, December 2007) using both emPCR kits II (primer A) and III (primer B) and sequenced on a GS FLX (Roche Diagnostics) by both primers on 1 lane/pool of a 16-lane gasket on a $70 \times 75$ FLX picotiterplate. The sequences from 1 lane were aligned to a backbone consisting of the merged PCR target regions derived from the human reference sequence (NCBI build 36.1, hg18, proximal gene cluster) and haplotype calls (HCs) were inferred using the GS Amplicon Variant Analyzer Software (Roche Diagnostics).

For verification, amplicon F5 (DEFB4) was generated from NA12760 and NA18502 by a separate PCR using the same fusion primers and conditions as described for the amplicon pools. GS FLX sequencing was done on 2 lanes/ amplicon of a 16-lane gasket on a $70 \times 75$ picotiterplate. To further increase sequencing depth, a third GS FLX run was performed with pooled amplicons F2, F4, F6 and F8 of NA12760, NA18502, NA18552, and NA18858 using a 4-lane gasket on a $70 \times 75$ picotiterplate. To avoid misinterpretations caused by incidental chimera formation early in PCR, 4 independent reactions were carried out with each primer pair and DNA sample.

\section{Amplicon subcloning in bacteria and Sanger sequencing} Using 7 out of the 11 DNAs (C0140, C0766, C0913, NA12760, NA18502, NA18858, NA19140), 2 PCR products (S1 and S2 [16]) were generated using unmodified primers (Metabion, Additional file 1, Table S1). PCRs were performed with 50 ng DNA using Taq polymerase (BIORAD). Amplification was achieved using 5 cycles of $95^{\circ} \mathrm{C}$ for $30 \mathrm{~s}, 56^{\circ} \mathrm{C}$ for $30 \mathrm{~s}$ and $72^{\circ} \mathrm{C}$ for $1 \mathrm{~min}$ followed by 30 cycles of $95^{\circ} \mathrm{C}$ for $30 \mathrm{~s}, 58^{\circ} \mathrm{C}$ for $30 \mathrm{~s}$ and $72^{\circ} \mathrm{C}$ for 1 min, and finally $72^{\circ} \mathrm{C}$ for $5 \mathrm{~min}$. PCR products were subcloned into PCR2.1-TOPO vector (Invitrogen) and 192 individual clones per DNA and amplicon were sequenced by dye terminator sequencing chemistry using an ABI3730xl automated sequencer (Applied Biosystems) and M13rev universal primers. Haplotypes were inferred by manual inspection of Phrap alignments using GAP4 (Staden package).

\section{Other copy number measurement methods}

The pyrosequencing based PRT (PPRT), the MLPA analyses [28] and the 5-PRT [18] were carried out as previously described. Results of CNs measured by $\mathrm{MAPH} /$ 
REDVR and another PRT [36] were kindly provided by J. Armour and E. Hollox.

\section{Results}

Haplotyping by 454 amplicon sequencing and comparison with the cloning/Sanger approach

The amplicons used in the present study were designed in order to cover a broad spectrum of regions within DEFB encompassing putative promoters, exons and introns and intergenic parts. If possible, amplicon lengths were restricted to span not much more than $250 \mathrm{bp}$ in order to obtain consistent haplotype information by 454 reads from both ends. Furthermore, the amplified regions were selected to contain a high number of known MSVs. Application of these criteria resulted in 8 amplicons (F1 to F8) with lengths of $194-340 \mathrm{bp}$, spread over a region of $87 \mathrm{~kb}$ (Fig. 1) and harbouring 32 known MSVs (31 single nucleotide exchanges and one single base insertion/deletion, dbSNP build 130, Additional file 1, Table S2). Each amplicon harbours between 2 (F2) and 6 MSVs (F8). Eleven genomic DNAs were selected to represent a broad range of CNs as determined by MLPA, that is, 2-9 DEFB copies per diploid genome. PCRs were performed separately for each DNA and primer pair followed by equimolar pooling of all PCR products of the same DNA. For validation purposes, 2 additional amplicons from 7 samples were analysed by cloning/Sanger sequencing [16] (S1: 4 MSVs/500 bp, S2: 5 MSVs/529 bp; Fig. 1, Additional file 1, Table S2).

Eleven lanes of a single GS FLX run (one amplicon pool per $1 / 16^{\text {th }}$ plate) resulted in 145,836 reads with an average length of $225 \mathrm{bp}$, producing a total of $32.8 \mathrm{Mb}$ raw data. Of the total reads, 142,033 reads (97.4\%) could be assembled to the amplicon reference sequences resulting in an average of 12,912 reads per DNA sample and 18,230 per amplicon (Additional file 1, Table S3). Among the amplicons, F1 and F3 were underrepresented by 6,955 and 8,082 reads, respectively, compared to $15,003-24,723$ for the remaining 6 amplicons. Additionally, the amount of reads per DNA sample greatly differed for F1 and F3 with less than 100 reads in 4 cases. Therefore, we have excluded the data of F1 and F3, leaving a total of 126,996 reads from 6 amplicons used for further analyses of the 11 DNA samples.

Every read may be regarded as representation of a particular DEFB allele. A haplotype can be inferred from each read by determination of the MSV allele combination, named "haplotype call" (HC). As amplicons were designed in accordance with the read length achievable by the GS FLX chemistry ( $250 \mathrm{bp}$ ), the small minority of reads greatly shorter than 250 bp produced by the FLX sequencing may not span the aspired haplotype. Such shorter HCs, however, are less informative and would complicate the analyses. Therefore, these suboptimal
HCs were omitted from further analyses, reducing the final data set from 126,996 reads to 123,003 most informative HCs (Additional file 1, Table S4).

Out of the 24 known MSVs within the 6 amplicons, 23 were found to be polymorphic. For rs4840825 (MSV16, amplicon F7), exclusively the $\mathrm{T}$ allele was observed. In addition, we confirmed in amplicon F5 of C0140, NA18858 and NA19140 an MSV at position 31 downstream of DEFB4 (A/G; chr8:7,259,758 and 7,791,677; MSV9a) not annotated in dbSNP but present in the hg18 reference sequence as a paralogous sequence variation [31]. Moreover, we identified a novel base exchange in the same amplicon of NA18502 located 24 nucleotides further downstream (C/A; chr8: 7,259,734 and 7,791,701; MSV10a).

The MSV combinations in 5 out of 6 amplicons could be unambiguously deduced from the overlapping forward and reverse reads. However, for F5 (340 bp, MSVs 6-10a) the read length of the FLX chemistry was too short to directly infer complete haplotypes, as the forward reads cover MSVs 6-8 and the reverse reads 7-10a, respectively (Table 1). Nevertheless, using data from non-polymorphic samples, the $\mathrm{HC}$ ratios in polymorphic samples and a parsimony assumption, 4 forward and 7 reverse haplotypes collapse consistently into 7 complete F5 haplotypes (Additional file 1, Table S5).

In total, 22 haplotypes could be identified (Table 1, Additional file 1, Table S5). The largest variety was found for amplicon F5 with 7 haplotypes out of 128 theoretically possible combinations of MSVs. In contrast, only 2 haplotypes were observed for F7 (8 theoretical haplotypes) and F8 (64), respectively. The most abundant haplotypes are for F2: GA, F4: TAC, F5: TCCGGGC, F6: CCTCG, F7: TCG and F8: CTACCG,. TCCGGGC and TCG were found in all investigated DNAs. Joining the haplotypes with the most HCs for each DNA sample individually creates 4 inferred "long range haplotypes" of which one is present in of 6 samples (Additional file 1, Table S6). Remarkably, this inferred haplotype can be generated by just one recombination of physical haplotypes represented by large insert clones from the RPCI-11 Human Male BAC Library, sequenced in the course of the human genome project [16] (Fig. 2).

Several additional MSV combinations were found which are represented by only few reads compared to the haplotypes described above, indicating either the generation of chimeric, PCR-mediated recombinant molecules from different target copies, misincorporations during the emulsion PCR, sequencing errors or cross-contaminations. In total, 53 out of 66454 PCRs and 10 out of 22 Sanger PCRs contained this phenomenon. Based on the estimation by alternative methods that the maximal DEFB CN in our sample set is 9, we discarded HCs with fractions $<0.10(1 / 10)$ as such artefacts. This led to the 
Table 1: Haplotype calls from 454 (run 1) and cloning/Sanger (CS) sequencing.

\begin{tabular}{|c|c|c|c|c|c|c|c|c|c|c|c|c|c|c|c|c|c|c|c|}
\hline \multirow{3}{*}{$\begin{array}{l}\text { Method } \\
\text { Amplicon } \\
\text { MSV HCs }\end{array}$} & \multirow{2}{*}{\multicolumn{2}{|c|}{$\begin{array}{c}454 \\
\text { F2 }\end{array}$}} & \multirow{3}{*}{$\begin{array}{l}\text { CS } \\
\text { S1 } \\
\#\end{array}$} & \multirow{2}{*}{\multicolumn{2}{|c|}{$\begin{array}{c}454 \\
\text { F5 }\end{array}$}} & \multirow{3}{*}{$\begin{array}{l}\text { CS } \\
\text { S2 } \\
\#\end{array}$} & \multirow{2}{*}{\multicolumn{2}{|c|}{$\begin{array}{c}54 \\
\text { F5 }\end{array}$}} & \multirow{3}{*}{$\begin{array}{l}\text { CS } \\
\text { S2 } \\
\#\end{array}$} & \multirow{2}{*}{\multicolumn{2}{|c|}{$\begin{array}{c}454 \\
\text { F4 }\end{array}$}} & \multirow{2}{*}{\multicolumn{2}{|c|}{$\begin{array}{c}454 \\
\text { F6 }\end{array}$}} & \multirow{2}{*}{\multicolumn{2}{|c|}{$\begin{array}{c}454 \\
\text { F7 }\end{array}$}} & \multirow{2}{*}{\multicolumn{2}{|c|}{$\begin{array}{c}54 \\
\text { F8 }\end{array}$}} & \multirow{2}{*}{\multicolumn{2}{|c|}{454}} \\
\hline & & & & & & & & & & & & & & & & & & & \\
\hline & $1-2$ & $\#$ & & $6-8$ & $\#$ & & $7-10 a^{*}$ & $\#$ & & $3-5$ & $\#$ & 11-15 & $\#$ & $16-18$ & $\#$ & $19-24$ & $\#$ & HCs & $\#$ \\
\hline \multirow[t]{3}{*}{ C0140 } & $\mathrm{GA}$ & 1.566 & 112 & $\mathrm{TCC}$ & 664 & 136 & CCGgGc & 1.491 & 81 & TAT & 582 & CCTCG & 867 & TCG & 888 & CTACCG & 542 & & \\
\hline & GG & 583 & 36 & & & & CCGaGc & 431 & 55 & CAT & 390 & CCTAG & 816 & TTA & 288 & TCTाTA & 195 & & \\
\hline & CA & 528 & 30 & & & & & & & TAC & 382 & & & & & & & 10.213 & 450 \\
\hline \multirow[t]{2}{*}{ C0766 } & GG & 1.525 & 114 & TCC & 360 & 140 & CCGgGc & 1.084 & 147 & TAC & 1.679 & & nd & TCG & 1.878 & CTACCG & 876 & & \\
\hline & $C A$ & 842 & 41 & $\mathrm{CCC}$ & 195 & 48 & CCGgAc & 506 & 41 & CAT & 601 & & & & & & & 9.546 & 531 \\
\hline \multirow[t]{2}{*}{ C0913 } & CA & 1.869 & 113 & $\mathrm{TCC}$ & 680 & 132 & CCGgGc & 1.221 & 145 & TAC & 1.556 & CCTCG & 1.195 & TCG & 1.816 & CTACCG & 2.153 & & \\
\hline & $\mathrm{GA}$ & 1.157 & 58 & $\mathrm{CCC}$ & 287 & 54 & CCGgAc & 617 & 41 & TCC & 634 & CCTAG & 593 & & & & & 13.778 & 543 \\
\hline \multirow[t]{3}{*}{ NA12760 } & $\mathrm{GA}$ & 1.750 & 92 & TCC & 795 & 99 & CCGgGc & 1.566 & 104 & TAC & 2.825 & CCTCG & 1.669 & TTA & 2.056 & TCTाTA & 645 & & \\
\hline & GG & 386 & 20 & $\mathrm{CCC}$ & 181 & 60 & CCGgAc & 339 & 55 & TAT & 981 & CTCAC & 599 & TCG & 1.696 & CTACCG & 605 & & \\
\hline & $C A$ & 319 & 19 & CTG & 184 & 0 & TGGgAc & 459 & 0 & $\mathrm{TCC}$ & 797 & CCTAG & 499 & & & & & 18.351 & 449 \\
\hline \multirow[t]{4}{*}{ NA15029 } & $\mathrm{GA}$ & 1.530 & & TCC & 767 & nd & CCGgGc & 1.046 & nd & $\mathrm{TCC}$ & 1.156 & CCTGC & 1.785 & TTA & 1.689 & TCTाTA & 377 & & \\
\hline & GG & 805 & & & & & CCAgGc & 431 & & TAT & 882 & CTCAT & 1.087 & TCG & 1.115 & CTACCG & 267 & & \\
\hline & & & & CTG & 475 & & TGGgAc & 913 & & TAC & 496 & & & & & & & & \\
\hline & & & & & & & & & & CAT & 420 & & & & & & & 15.241 & \\
\hline \multirow[t]{3}{*}{ NA15213 } & $\mathrm{GA}$ & 1.326 & & TCC & 665 & nd & CCGgGc & 1.338 & nd & TAC & 278 & CCTCG & 1.178 & TCG & 1.496 & CTACCG & 929 & & \\
\hline & $C A$ & 349 & & CTG & 171 & & TGGgAc & 390 & & TAT & 98 & CCTAG & 577 & TTA & 866 & TCTTTA & 551 & & \\
\hline & & & & & & & & & & TCC & 87 & CTCAT & 473 & & & & & 10.772 & \\
\hline \multirow[t]{3}{*}{ NA15385 } & $\mathrm{GA}$ & 985 & & TCC & 374 & nd & CCGgGc & 702 & nd & TAC & 1.374 & CCTCG & 1.003 & TCG & 938 & CTACCG & 688 & & \\
\hline & GG & 592 & & $\mathrm{CCC}$ & 169 & & CCGgAc & 239 & & TAT & 673 & CCTAG & 322 & TTA & 782 & TCTाTA & 483 & & \\
\hline & & & & CTG & 88 & & TGGgAc & 188 & & $\mathrm{TCC}$ & 345 & CTCAT & 280 & & & & & 10.225 & \\
\hline NA18502 & $\mathrm{GA}$ & 812 & 80 & $\mathrm{TCC}$ & 182 & 125 & CCGgGc & 265 & 139 & TAT & 1.453 & CCTCG & 415 & TTA & 856 & TCTITA & 645 & & \\
\hline
\end{tabular}


Table 1: Haplotype calls from 454 (run 1) and cloning/Sanger (CS) sequencing. (Continued)

\begin{tabular}{|c|c|c|c|c|c|c|c|c|c|c|c|c|c|c|c|c|c|c|c|}
\hline & $C A$ & 561 & 58 & $\mathrm{CCC}$ & 21 & 15 & CCGgGa & 56 & 0 & & & TTСAT & 65 & TCG & 418 & CTACCG & 267 & & \\
\hline & & & & CCG & (7) & 0 & CGGgAc & 39 & 0 & & & & & & & & & 5.995 & 402 \\
\hline \multirow[t]{3}{*}{ NA18552 } & CA & 3.490 & & TCC & 197 & nd & CCGgGc & 273 & nd & TAT & 225 & CCTCG & 1.653 & TCG & 1.756 & CTACCG & 1.525 & & \\
\hline & & & & $\mathrm{CCC}$ & 328 & & CCGgAc & 340 & & TAC & 1.058 & & & & & & & & \\
\hline & & & & & & & & & & CAT & 503 & & & & & & & 11.348 & \\
\hline \multirow[t]{3}{*}{ NA18858 } & $\mathrm{GA}$ & 677 & 97 & TCC & 1.234 & 188 & CCGgGc & 623 & 124 & TAT & 1.490 & CCTCG & 1.327 & TTA & 1.087 & TCTTTA & 2.404 & & \\
\hline & $C A$ & 324 & 45 & & & & CCGaGc & 218 & 51 & TAC & 822 & & & TCG & 404 & CTACCG & 633 & & \\
\hline & GG & 205 & (16) & & & & & & & & & & & & & & & 11.448 & 505 \\
\hline \multirow[t]{3}{*}{ NA19140 } & $\mathrm{GA}$ & 314 & 57 & $\mathrm{TCC}$ & 706 & nd & CCGgGc & 234 & nd & & nd & CCTCG & 504 & TTA & 281 & & nd & & \\
\hline & CA & 310 & 73 & & & & CCGaGc & 42 & & & & CTCAT & 130 & TCG & 62 & & & & \\
\hline & GG & 105 & 24 & & & & & & & & & & & & & & & 2.688 & 154 \\
\hline Total & & 22.910 & 1.069 & & 8.702 & 982 & & 15.012 & 983 & & 21.787 & & $\begin{array}{r}17.03 \\
7\end{array}$ & & 20.372 & & 13.785 & 119.605 & $\begin{array}{r}3.03 \\
4\end{array}$ \\
\hline
\end{tabular}

\#: number of $\mathrm{HCs} ;{ }^{*}$ : alleles in lower cases refer to the 2 additionally identified MSVs 9a and 10a; (): nominally below $10 \%$ threshold; nd: no data. 


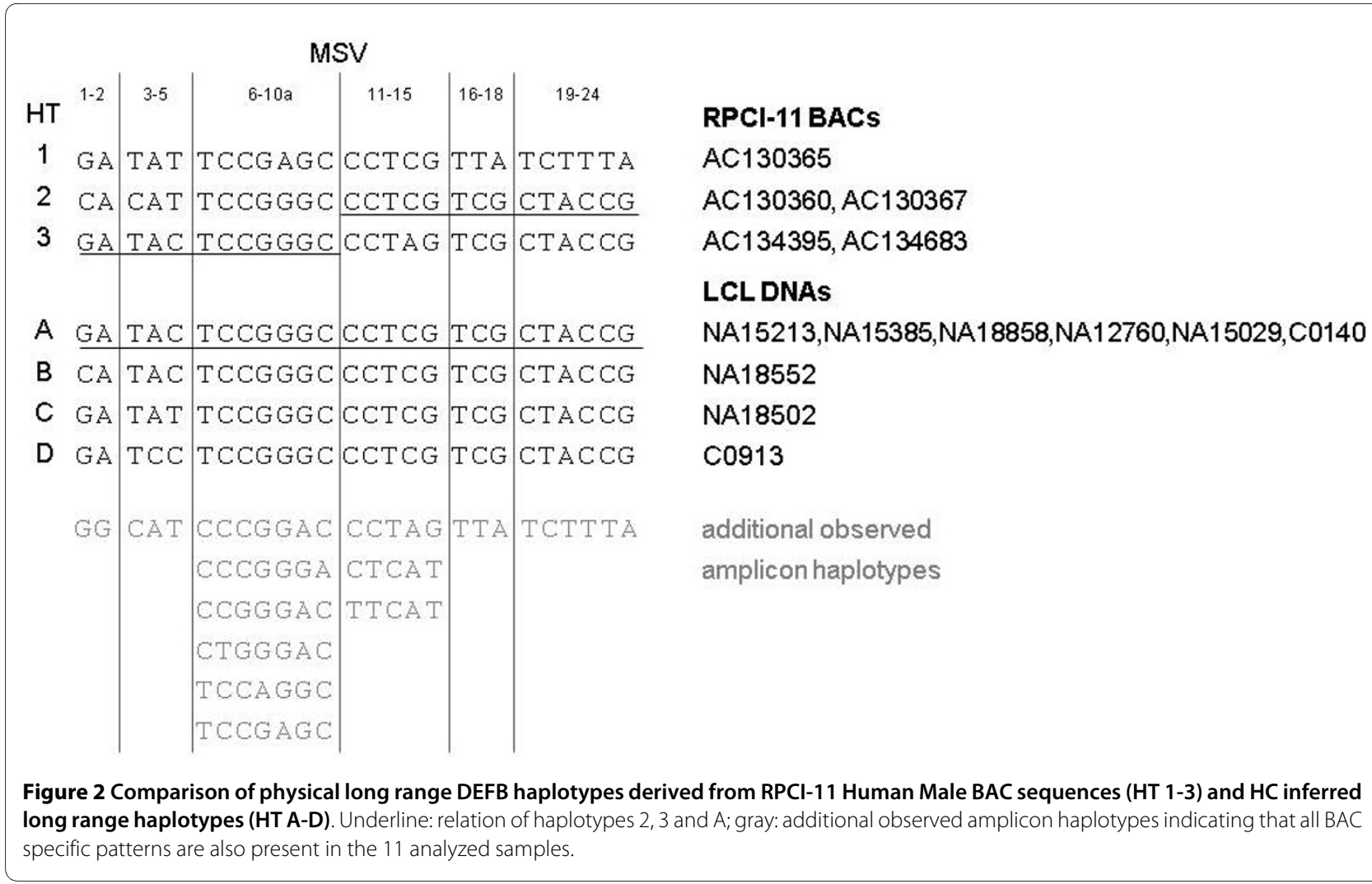

exclusion of 3,338 (2.7\%) and $86(2.7 \%)$ HCs from the 454 and Sanger datasets, respectively (Table 1, Additional file 1, Table S4).

Comparing the haplotyping results for both methods, the same haplotypes were found in $85 \%$ (11/13) except for amplicons F5/S2 in samples NA12760 and NA18502 (Table 1). In both cases rare 454 haplotypes were not found in the Sanger reads. Due to the low overall number of F5 sequences for NA18502, the HC frequency for the haplotype inferred from MSVs 6-8 is lower $<0.10$ and had to be excluded (Table 1, value in brackets; Additional file 1 , Table S4). In order to finally confirm or reject the questionable haplotypes, we generated additional F5 amplicons from the 2 DNA samples and performed 454 sequencing in $2 / 16^{\text {th }}$ plate per sample resulting in 33,305 and 33,205 sequences, respectively. From these reads more than 10,000 HCs per sample for MSVs 6-8 and over 20,000 calls for MSVs 7-10a were obtained for each of the 2 DNAs, unequivocally confirming the presence of the questionable CTGGGAC and CCGGGAC haplotypes, respectively (Table 2 ).

\section{Determination of relative DEFB copy numbers by haplotype calls}

In order to evaluate the applicability of $454 \mathrm{HCs}$ for the measurement of relative DEFB CNs, we calculated the
$\mathrm{HC}$ ratios for all amplicons with more than one haplotype and inferred the respective minimal $\mathrm{CN}$. For example, in F2 from C0140, 3 haplotypes (CA, GA, and GG) were identified with 528, 1,566 and 583 calls, respectively. This corresponds to a normalized call ratio of 1:2.97:1.10 that is essentially $1: 3: 1$, indicating a minimal $\mathrm{CN}$ of 5 . Altogether, by this approach $60 \mathrm{CN}$ calculations were done. For samples with more than one polymorphic amplicon we computed the average of the separate CNs or of their least common multiple. The final minimal $\mathrm{CN}$ estimations for the samples range between 3 and 9 (Table 3, Additional file 1, Table S7).

The DEFB CNs of the 11 samples haplotyped in this study were previously measured by 40 independent experiments using different PRTs, MLPA and MAPH/ REDVR [18,28,36] and Armour and Hollox, personal communication]. For 8 of the 11 DNAs, measurement by the different techniques (not including our) resulted in identical CNs. The remaining samples showed disagreements of only one copy, indicating the consistency of the data (Table 3, Additional file 1, Table S7). Therefore, we took the average of these measurements and compared them with $\mathrm{CN}$ estimates from the $454 \mathrm{HCs}$. For 7 samples the 454 haplotype-based estimates were in agreement with CNs determined by the alternative methods. For another 2 DNA samples (NA15213, NA15385), these 
Table 2: Haplotype calls of ultra-deep amplicon F5 454 sequencing (run 2) for NA12760 and NA18502.

\begin{tabular}{|c|c|c|c|c|c|c|}
\hline \multirow[b]{2}{*}{ MSV HCs } & \multicolumn{3}{|c|}{ F5 forward } & \multicolumn{3}{|c|}{ F5 reverse } \\
\hline & $6-8$ & $\#$ & $f$ & 7-10a* & $\#$ & f \\
\hline \multirow[t]{3}{*}{ NA12760 } & TCC & 7.399 & 0,69 & CCGgGc & 14.683 & 0,72 \\
\hline & $\mathrm{CCC}$ & 1.765 & 0,16 & CCGgAc & 2.891 & 0,14 \\
\hline & CTG & 1.591 & 0,15 & TGGgAc & 2.895 & 0,14 \\
\hline Total & \multicolumn{3}{|c|}{10.755} & \multicolumn{3}{|c|}{20.469} \\
\hline \multirow[t]{3}{*}{ NA18502 } & TCC & 8.307 & 0,80 & CCGgGc & 17.586 & 0,83 \\
\hline & $\mathrm{CCC}$ & 1.069 & 0,10 & CCGgGa & 1.764 & 0,08 \\
\hline & CCG & 1.006 & 0,10 & CGGgAc & 1.950 & 0,09 \\
\hline Total & \multicolumn{3}{|c|}{10.382} & \multicolumn{3}{|c|}{21.300} \\
\hline
\end{tabular}

\#: number of $\mathrm{HCs} ; \mathrm{f}=\mathrm{HC}$ frequency; ${ }^{*}$ : alleles in lower cases refer to the 2 additionally identified MSVs $9 \mathrm{a}$ and $10 \mathrm{a}$.

numbers differed only by one copy. In the case of NA18858, the estimated minimal CN was half of the absolute one. For NA18552, 3 MSV combinations are polymorphic, resulting in CNs of 5 (2:3), $2 \mathrm{n}$ (1:1) and 7 (2:4:1), respectively. Only in this single case, the $\mathrm{HC}$ based minimal $\mathrm{CN}$ estimations can not be unambiguously interpreted and considerably deviates from 2 copies per diploid genome, consistently determined by 4 alternative methods.

As ultra-deep sequencing of NA12760 and NA18502 amplicon $\mathrm{F} 5$ provided most consistent $\mathrm{CN}$ estimations (Table 2, Additional file 1, Table S7) for samples with high CNs, we further validated this approach by performing a third GS FLX run with pooled amplicons F2, F4, F6 and F8 from these DNAs. Moreover, we included NA18552 $(\mathrm{CN}=7$ by $\mathrm{HCs}$ of amplicons F4) and NA18858 (CN $=8$ by alternative methods) (Table 3 ). To avoid false positive HCs resulting from chimera formation early in PCR, we carried out 4 independent reactions with each primer pair and DNA sample. Accordingly, we lowered the threshold for exclusion of minor haplotype fractions from $10 \%$ to $6 \%$ to avoid false negative HCs. Altogether, we obtained 405,574 HCs providing on average $\sim 25,000$ per amplicon and sample (Additional file 1, Table S8), of which $1.4 \%$ were excluded as potential chimera, sequencing errors or contaminations. The extraordinary sequencing depth and the lowered filter threshold identified additional haplotypes for NA18502 (F2: GG) and NA18858 (F4: CAT, F6: TTCAT) which increased the resolution of the CN estimation (Table 4, Additional file 1, Table S9). Ultra-deep sequencing-based $\mathrm{CN}$ estimations for samples with high CNs (NA18502, NA18858) tend to be higher than those determined by the established alternative methods.

\section{Discussion}

Impaired beta-defensin synthesis has been described in many human diseased states, namely in inflammatory disorders [20,22,29,30,42-44]. While association of a disease and DEFB CN has been demonstrated [20,29,30], associations of sequence variants with disease [18] cannot reliably be investigated with established genotyping methods. Hollox [22] observed that it may be the nucleotide state of an MSV that actually causes susceptibility to a disease for which association with $\mathrm{CN}$ has been found. If so, $\mathrm{CN}$ is only a proxy for causative sequence variants. However, description of sequence variation in $\mathrm{CN}$ variable loci such as DEFB at 8 p23 is inherently challenging. Currently, neither the haplotype structure of DEFBs nor their arrangements on chromosomes (DEFB-locus alleles) are known. The present work provides a first glimpse of the DEFB haplotype complexity through 454 amplicon sequencing of selected polymorphic defensin gene fragments. Among the currently available next generation sequencing (NGS) approaches, the 454 technology is best suited for this purpose as it provides longest reads that in turn produce the longest inferred physical haplotypes.

Eleven DNA samples derived from LCLs were selected to represent a range of 2-9 DEFB copies per diploid genome. All DNAs are publicly available and 4 were investigated in the framework of the HapMap project [40]. Four of the LCLs were established from individuals of Caucasian, 3 of Yoruba and one of Chinese ancestry. About 120,000 MSV HCs were derived from 6 amplicons containing 2-6 annotated MSVs each. One MSV not deposited in dbSNP and a new one were detected in 3 samples of Yoruba and one of Caucasian ancestry, respectively. Theoretically, the 26 MSVs in 6 amplicons alto- 
Table 3: DEFB copy numbers per diploid genome estimated by the ratio of 454 haplotype calls (run 1) in comparison to alternative methods.

\begin{tabular}{|c|c|c|c|c|c|c|c|c|c|c|c|c|c|c|c|c|}
\hline \multirow{3}{*}{$\begin{array}{c}\text { Amplicon } \\
\text { MSVs }\end{array}$} & \multicolumn{7}{|c|}{454} & \multicolumn{9}{|c|}{ Alternative methods } \\
\hline & \multirow{2}{*}{$\begin{array}{l}F 2 \\
1-2\end{array}$} & \multirow{2}{*}{$\begin{array}{l}F 5 f \\
6-8\end{array}$} & \multirow{2}{*}{$\begin{array}{c}\text { F5r } \\
7-10 a\end{array}$} & \multirow{2}{*}{$\begin{array}{l}F 4 \\
3-5\end{array}$} & \multirow{2}{*}{$\begin{array}{c}\text { F6 } \\
11-15\end{array}$} & \multirow{2}{*}{$\begin{array}{c}\text { F7 } \\
16-18\end{array}$} & \multirow{2}{*}{$\begin{array}{c}\text { F8 } \\
19-24\end{array}$} & \multicolumn{2}{|l|}{ Average } & \multirow[t]{2}{*}{ Armour } & \multirow[t]{2}{*}{ 5PRT } & \multirow[t]{2}{*}{ PPRT } & \multirow[t]{2}{*}{ MLPA } & \multicolumn{2}{|c|}{ Average } & \multirow{2}{*}{$\begin{array}{c}\text { 454-Altern. } \\
\sim\end{array}$} \\
\hline & & & & & & & & & $\sim$ & & & & & & $\sim$ & \\
\hline Co140 & 5 & & 4 & & $4(2 n)$ & 4 & 4 & 4.20 & 4 & 4 & 3.78 & 4.19 & 4 & 3.99 & 4 & 0 \\
\hline C0766 & 3 & 3 & 3 & 4 & & & & 3.25 & 3 & 3 & 2.75 & 3.31 & 3 & 3.01 & 3 & 0 \\
\hline C0913 & 3 & 3 & 3 & 3 & 3 & & & 3.00 & 3 & 3 & 3.00 & 3.13 & 3 & 3.03 & 3 & 0 \\
\hline NA12760 & 7 & 6 & 6 & 6 & 5 & $6(2 n)$ & $6(2 n)$ & 6.00 & 6 & 6 & 5.57 & 6.04 & 6 & 5.90 & 6 & 0 \\
\hline NA15029 & $6(3 n)$ & 5 & 5 & 6 & 5 & 5 & 5 & 5.29 & 5 & nd & 4.70 & 5.58 & 5 & 5.09 & 5 & 0 \\
\hline NA15213 & 5 & 5 & 4 & 5 & 4 & 5 & 5 & 4.71 & 5 & nd & 4.18 & 4.18 & 4 & 4.12 & 4 & +1 \\
\hline NA15385 & 5 & 7 & 5 & 7 & 5 & $6(2 n)$ & 5 & 5.71 & 6 & nd & 5.29 & 5.17 & 5 & 5.15 & 5 & +1 \\
\hline NA18502 & 10 & 10 & 9 & & 7 & $9(3 n)$ & $8(4 n)$ & 8.83 & 9 & 8 & 8.98 & nd & 9 & 8.66 & 9 & 0 \\
\hline NA18552 & & 5 & $6(2 n)$ & 7 & & & & 6.00 & 6 & 2 & 2.23 & 2.02 & 2 & 2.06 & 2 & +4 \\
\hline NA18858 & 4 & & 4 & 3 & & 4 & 5 & 4.00 & 4 & 8 & 7.71 & 8.08 & 8 & 7.95 & 8 & -4 \\
\hline NA19140 & 7 & & 7 & & 5 & 6 & & 6.25 & 6 & 7 & 5.71 & 6.26 & 6 & 6.24 & 6 & 0 \\
\hline
\end{tabular}

Armour: PRT and MAPH/REDVR [36] and personal communication; 5PRT. PPRT [18,28]; MLPA [28]. : rounded; n: multiple of the minimal estimated CN; *: CN estimations based on ultra-deep 454 sequencing; nd: no data; blank: non polymorphic. 
Table 4: DEFB copy numbers per diploid genome estimated by 454 ultra-deep sequencing (run 2: F5; run 3: F2, F4, F6, F8) in comparison to alternative methods.

\begin{tabular}{|c|c|c|c|c|c|c|c|c|c|}
\hline \multirow[b]{2}{*}{ Amplicon } & \multicolumn{6}{|c|}{454} & & \multirow{2}{*}{$\begin{array}{c}\text { Alternative } \\
\text { methods } \\
\text { Average }\end{array}$} & \multirow[t]{2}{*}{ 454-Altern } \\
\hline & F2 & F5f & F5r & F4 & F6 & F8 & Average & & \\
\hline NA12760 & 8 & 7 & 7 & 7 & 5 & 9 & 7 & 6 & +1 \\
\hline NA18502 & 11 & 10 & 11 & & 9 & 10 & 10 & 9 & +1 \\
\hline NA18552 & & nd & nd & 8 & & & 8 & 2 & +6 \\
\hline NA18858 & 10 & nd & nd & 12 & 11 & 10 & 11 & 8 & +3 \\
\hline
\end{tabular}

nd: no data; blank: non polymorphic.

gether could compose 268 haplotypes of which we identified $22(8 \%)$. As exemplified by the analysis of the F5 amplicon data, HC ratios provided by the 454 deep sequencing approach may serve as additional experimental evidence to deduce longer haplotypes than the maximal NGS read length.

With 26 MSVs in 6 amplicons, totalling to $1,498 \mathrm{bp}$, the MSV density in the investigated regions is approximately $17 \mathrm{MSV} / \mathrm{kb}$. This is higher than the overall single nucleotide variation density of the entire DEFB currently annotated in dbSNP $(2,971 \mathrm{MSVs} / 229 \mathrm{~kb}=13 \mathrm{MSV} / \mathrm{kb})$ and reflects the directed selection of the amplified regions to screen a maximum of polymorphic positions within a short distance. In comparison, for the most sequence variable locus of the human genome, the MHC complex, alignments of $8 \mathrm{MHC}$ haplotypes revealed $8 \mathrm{SNP} / \mathrm{kb}$ $(37,451 \mathrm{SNPs} / 4.7 \mathrm{Mb})$ with a higher density $(13 \mathrm{SNP} / \mathrm{kb})$ in the intragenic regions [45]. For the genomic loci of HLA-A, B and C there are 1,578 polymorphic positions within 10,020 bp assigned by dbSNP build 130, corresponding to $157 \mathrm{SNPs} / \mathrm{kb}$. This indicates that in terms of sequence variability, DEFB is substantially less polymorphic than the HLA loci, and that DEFB together with the analysed amplicons are comparable to the MHC intragenic regions. The fact that the 454 deep-sequencing revealed only one novel MSV on the background of 24 previously known ones shows that the dbSNP annotation of the analysed regions is quite comprehensive. On the other hand according to dbSNP, the MSV density of DEFB is about 4 times higher than the SNP density of the entire human genome (4 SNP/kb). Moreover, currently unknown additional sequence variability may be still hidden in the DEFB (e.g. $25 \mathrm{MSV} / \mathrm{kb}$ upstream of DEFB4 observed in 16 LCLs; Groth, unpublished) which may be revealed by future targeted and whole-genome resequencing efforts.

The haplotypes identified by 454 sequencing and by cloning/Sanger sequencing were identical except for 2 cases, which could be resolved by additional 454 sequencing. However, the ratios of haplotypes derived from a given individual amplicon sometimes differ remarkably. For example, the C0913 F5 haplotypes CCGGAC and CCCGGC were revealed by 454 sequencing in a ratio of 1:2 (617/1,221 calls) but 1:3.5 (41/145 calls) by Sanger derived reads. We suspect the explanation is that the number of HCs from Sanger sequenced clones is too low for providing reliable results. Moreover, additional bias may be introduced e.g. by the bacterial cloning step.

Theoretically, the more HCs that are available, the more accurately they should reflect the real ratio of the DEFB copies with particular haplotypes. This becomes more important as the number of DEFBs and/or haplotypes per genome increases. The conclusion is supported by our efforts to resolve the discrepancies between 454 and Sanger sequencing for NA12760 and NA18502. The minor haplotypes of the F5 amplicon were supported by 454 but not by Sanger sequencing, probably due to the low amount of sequenced clones. Additional $454 \mathrm{HCs}$ finally confirmed the presence of the F5 haplotypes under question.

Furthermore we noticed an uneven distribution of forward and reverse reads in the 454 sequencing of particular amplicons. This is obvious in Fig. 3 with a nearly 1:2 forward-to-reverse read ratio and is also reflected in the entire data set of amplicon F5 with 8,702 forward but 15,020 reverse HCs (1:1.7). Such a skew may be caused by unequal sequencing efficiencies due to the direction-specific sequencing primers as well as to differences in base composition and/or motifs between the forward and reverse strands. Thus in the case of non or partially overlapping forward and reverse reads, the resolution of the approach is limited by the less effective sequencing direction $[46,47]$.

Another problem of any PCR-based haplotyping is the occurrence of chimeric products [32]. Since both 454 amplicon sequencing and the cloning/Sanger approach are based on PCR, the formation of such artefacts must be taken into account. Chimeras can be generated during 
PCR by heteroduplexes of e.g. a complete copy of one allele with a fragment from another allele. Completion of the partial strand in the elongation step creates chimeric molecules from which chimeric haplotypes may be erroneously determined. The degree of chimera formation is dependent on various factors such as polymerase, nucleotide/primer concentrations, cycling conditions and amplicon length. In addition to the formation of chimeric PCR products, nucleotide misincorporations during the emulsion PCR, sequencing errors and cross-contaminations have to be taken into account. In the current study, we have appointed and discarded an MSV combination as artificial if its relative number of call was lower than $10 \%(1 / 10$; cloning/Sanger and GS FLX run 1$)$ or $6 \%(1 /$ 16; ultra-deep FLX runs 2 and 3). This led to the exclusion of less than $3 \%$ of all HCs which otherwise would render the analysis impossible. False negative HCs by this cutoff per definitionem would only affect DNA samples with more than 10 and 16 DEFB copies, respectively. CNs $>10$ are rarely reported in the literature $[23,29,30]$. Nevertheless, these threshold filters may fail if the artificial molecule appears early in PCR. To avoid respective misinterpretations, independent PCRs were carried out and pooled before sequencing for the third GS FLX run.

The estimation of CNs from the $\mathrm{HC}$ ratios is intrinsically problematic, since ratios provide only minimal $\mathrm{CNs}$ and any multiple of that can be ruled out only by addi- tional considerations (NA18858). Moreover, slightly different ratios lead to different $\mathrm{CN}$ estimations. Nevertheless, thanks to the high number of NGS HCs, CNs could be correctly determined for 7 out of 11 samples and differed only by one copy per diploid genome for another 2 samples (Table 3, Additional file 1, Table S7). Remarkably, the high $\mathrm{HC}$ numbers obtained by ultradeep GS FLX runs 2 and 3 consistently point towards higher CNs than obtained by the widely used PRTs/ MPLA (Tab. 4, Additional file 1, Table S9). It is known that the latter methods tend to underestimate the actual $\mathrm{CN}$ in case of DEFB CNs $>5$ (Huse, unpublished). Thus, it has to be proven in the future, whether ultra-deep NGS like 454 may provide more reliable results than the currently established methods for cases of high CNs per diploid genome. The recently developed mrFAST (microread fast alignment search tool) [48] points in the same direction although the sequencing depth of personalized whole-genome sequencing efforts and the 1000 Genomes Project is too low to provide sufficient read counts for reliable estimations of high $\mathrm{CNs}$ in complex regions like DEFB.

Only in one case were the $\mathrm{CN}$ estimations inconsistent (NA18552: 5-8 copies by 454 sequencing $v s .2$ copies by 4 alternative methods, Tables 3 and 4). For this, we suppose 2 possible reasons. First, the estimated CN 5 results from amplicon F5 with a relatively low amount of reads which

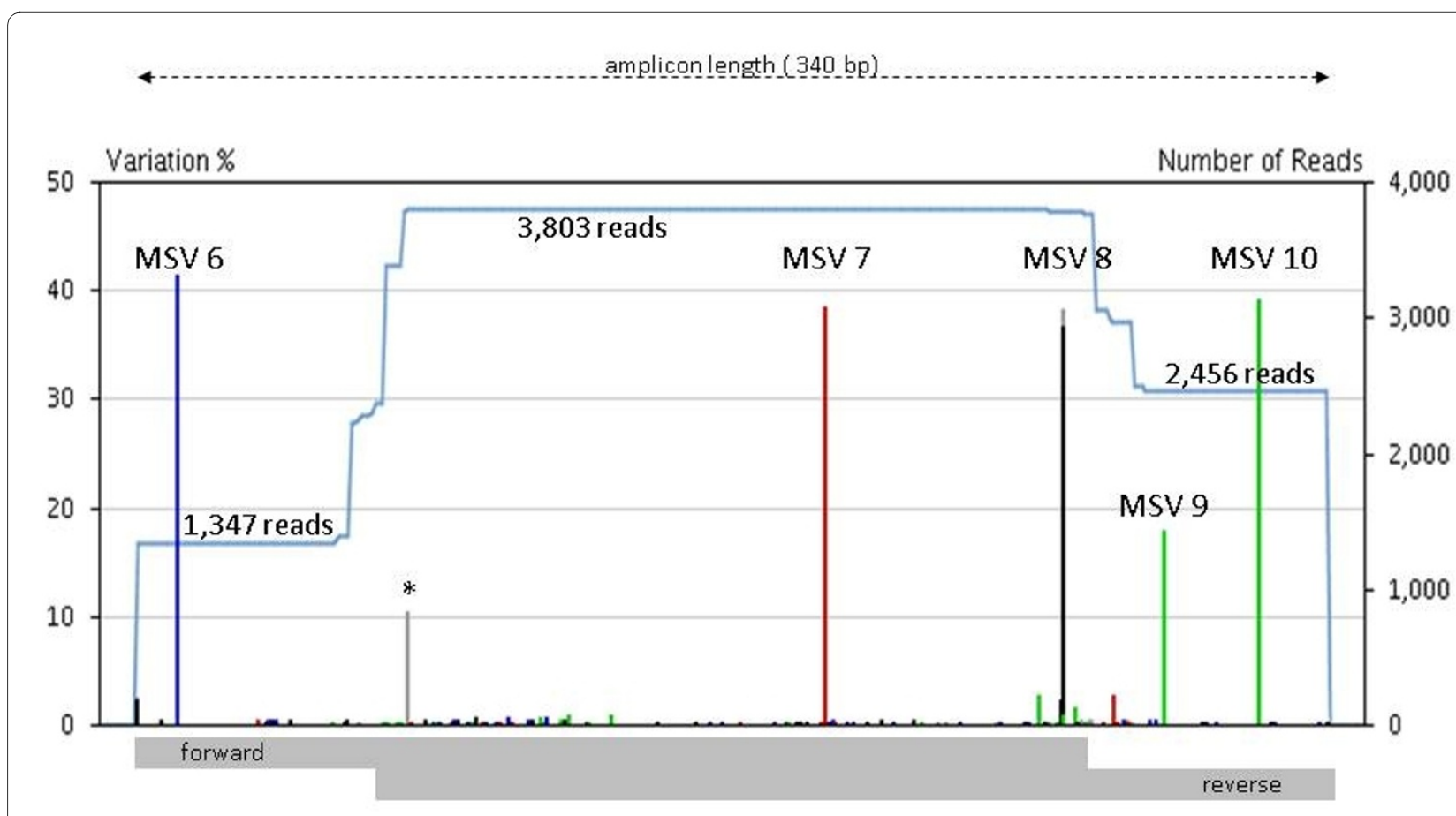

Figure 3 Graphical output of the GS Amplicon Variant Analyzer for the assembly of sequences as exemplarily shown for NA15029, amplicon F5. Left Y-axis: minor allele frequency; MSV colour code: blue =C; red = T; black= G; green = A; asterisk: insertion/deletion 454 sequencing artefact within a poly-A stretch; blue curve: read coverage; grey bar below: average lengths of forward and reverse reads, respectively. 
in case of the forward sequencing direction (MSVs 6-8) caused a random deviation from the 1:1 ratio observed for the reverse HCs. Second, 7-8 copies were calculated by the ratio of 3 haplotypes in amplicon F4. As the 3 haplotypes were confirmed by ultra-deep sequencing of pooled independent amplicons, it is unlikely that the minor haplotype (TAT) is a chimera. As the remaining 454 amplicons show no sequence variation and are therefore in agreement with the low $\mathrm{CN}$ of 2 determined by the alternative methods, sample NA18552 may represent in our hands the first example violating the concordance rule of DEFB [28]. Whether the underlying molecular event represents a natural polymorphism or emerged in cell culture remains to be elucidated in the future.

Although our results indicate that NGS based haplotyping and $\mathrm{CN}$ estimation thereof is a suitable approach to characterize a highly polymorphic and $\mathrm{CN}$ variable loci as the DEFB cluster, this method has some inherent limitations. First, haplotyping is restricted to those regions in which at least two MSVs are located within the read length of the applied NGS technology. Second, equimolar pooling of PCR products is difficult and also the efficiencies of the following emulsion PCR may deviate among the amplicons. This can result in differing amounts of HCs per amplicon which may prevent reliable haplotyping if too few sequences are obtained (e.g. from our amplicons F1 and F3). Third, formation of PCR artefacts leading to the identification of "false", i.e. chimeric haplotypes always has to be considered. Although distinction between "true" and "false" halpotypes is easier with higher sequencing depth it is problematic for samples with high CNs.

\section{Conclusion}

Deep 454 amplicon sequencing is an effective method for parallel haplotyping and $\mathrm{CN}$ estimation in highly polymorphic loci such as DEFB at chromosome 8p23.1 for the following reasons:

1. There is no bacterial cloning bias as a source of putative errors in haplotype identification. Moreover, in contrast to the cloning/Sanger sequencing approach, thousands of HCs per amplicon can easily be performed leading to a higher accuracy in obtained haplotype ratios and $\mathrm{CN}$ estimations inferred thereof.

2. Calculated on the basis of the consumable's prices for the present study and normalized to the same yield of informative $\mathrm{HCs}$, the costs for 454 amplicon sequencing amounts to only $\sim 4 \%$ of those for cloning/Sanger sequencing. Although this does not even consider the reduced experimental effort it is still too expensive for high-throughput measurements but acceptable for analyses of small cohorts.

3. Recently, 454 Titanium amplicon sequencing protocols and kits became available (Roche Diagnostics), increasing achievable read lengths to $400 \mathrm{bp}$ with a 2-fold increase of the number of reads per run. Thus, longer amplicons than in our study can be sequenced, encompassing more or more distant MSVs. Moreover, as the output of reads also increases, multiplexing different DNAs will be feasible by application of barcoding strategies (multiplex identifier, MID).

4. Although the presented 454 amplicon sequencing delivers only relative $\mathrm{CNs}$ and has to be completed by other approaches, future inclusion of reference amplicons from loci with invariable $\mathrm{CN}$ will provide absolute DEFB CNs.

Deep 454 amplicon sequencing can contribute substantially to investigate sequence variants and haplotype structure of $\mathrm{CN}$ variable loci. Nevertheless, increases in both NGS read length and output are needed to eventually completely resolve loci like DEFB at the physical haplotype level.

\section{Additional material}

\begin{abstract}
Additional file 1 Amplicons ( $F=G S F L X / S=$ Sanger sequencing), primers and their locations in the human genome. PCR products (amplicons), primers used for their amplification and chromosomal locations (NCBI build 36.1, hg18) of the amplified regions. MSVs and amplicons. Analyzed multisite variations (MSVs), their rs numbers and chromosomal locations according to SNPdb build 130 and NCBI build 36.1 (hg18), respectively. Numbers of assembled GS-FLX reads per amplicon (F1 to F8) and DNA (run1 and run 2). Assembled 454 sequences from 2 GS FLX runs per DNA and amplicon. Haplotypes and haplotype calls (HCs) for GSFLX runs 1-2 and PCR/cloning. Haplotypes, haplotype calls (HCs) and their fraction per DNA and MSV combination, derived from GS FLX runs 1 and 2 and Sanger sequencing of cloned PCR products. Haplotypes (HTs) by MSV combinations. Haplotypes and total number and fraction of haplotype summed up from all DNAs sequenced in GS FLX runs 1 and 2. Haplotypes by DNA. Identified haplotypes per DNA and amplicon, highlighting the most abundant haplotypes. Individual DEFB copy numbers (CN) derived from the ratio of haplotype calls (HCs) per MSV combination and comparison with $\mathrm{CNs}$ by other methods. Estimation of of the DEFB cluster copy number (CN) from the ratio of haplotype calls ( $\mathrm{HCS}$ ) per DNA and amplicon and comparison of with CNs determined by MLPA and different paralogue ratio tests. Haplotypes and haplotype calls (HCs) for GS-FLX run 3. Haplotypes, haplotype calls (HCs) and their fraction per DNA and MSV combination, derived from GS FLX run 3 (ultra-deep sequencing). Individual DEFB copy numbers (CN) derived from the ratio of haplotype calls (HCs) per MSV combination from run 1+2 versus run3. Individual DEFB copy numbers (CN) for 4 DNAs in comparison between GS FLX run $1+2$ versus GS FLX run 3 (ultra deep sequencing).
\end{abstract}

\section{Authors' contributions}

ST designed the 454 experiments and carried out both the 454 and Sanger experiments, designed by KH. MG performed the MLPA and PRT experiments. ST, AP and KS analyzed the data. MP, ST, JH, PR and SS conceived of the study, and participated in its design and coordination. ST, MP, KH and KS wrote the manuscript. All authors read and approved the final manuscript.

\section{Acknowledgements}

We thank Daniela Werler, Cornelia Luge, Silke Foerste and Beate Szafranski for excellent technical assistance. John Armour and Ed Hollox are acknowledged for kindly providing CN data based on MAPH/REDVR and PRT. We thank Niels Jahn for bioinformatics support as well as Roger Horton and Andrew Heidel for critically reading the manuscript. This work was supported by grants from the Bundesministerium für Bildung und Forschung (01GS0809, 01GS08182) and the GP9 project of the Network of Environmental Disorders. 


\section{Author Details}

'Leibniz Institute for Age Research - Fritz Lipmann Institute, D-07745 Jena, Germany, Institute of Clinical Molecular Biology, Christian-Albrechts-University D-24105 Kiel, Germany and '3Dept. of General Intermal Medicine, ChristianAlbrechts-University D-24105 Kiel, Germany

Received: 22 January 2010 Accepted: 19 April 2010 Published: 19 April 2010

\section{References}

1. Margulies M, Egholm M, Altman WE, Attiya S, Bader JS, Bemben LA, Berka J, Braverman MS, Chen YJ, Chen Z, Dewell SB, Du L, Fierro JM, Gomes XV, Godwin BC, He W, Helgesen S, Ho CH, Irzyk GP, Jando SC, Alenquer ML, Jarvie TP, Jirage KB, Kim JB, Knight JR, Lanza JR, Leamon JH, Lefkowitz SM, Lei $\mathrm{M}$, Li J, et al:: Genome sequencing in microfabricated high-density picolitre reactors. Nature 2005, 437(7057):376-380.

2. Bontell IL, Hall N, Ashelford KE, Dubey JP, Boyle JP, Lindh J, Smith JE: Whole genome sequencing of a natural recombinant Toxoplasma gondii strain reveals chromosome sorting and local allelic variants. Genome Biol 2009, 10(5):R53.

3. Diguistini S, Liao NY, Platt D, Robertson G, Seidel M, Chan SK, Docking TR, Birol I, Holt RA, Hirst M, Mardis E, Marra MA, Hamelin RC, Bohlmann J, Breuil C, Jones SJ: De novo genome sequence assembly of a filamentous fungus using Sanger, 454 and Illumina sequence data. Genome Biol 2009, 10(9):R94.

4. Novo M, Bigey F, Beyne E, Galeote V, Gavory F, Mallet S, Cambon B, Legras $J$, Wincker P, Casaregola S, Dequin S: Eukaryote-to-eukaryote gene transfer events revealed by the genome sequence of the wine yeast Saccharomyces cerevisiae EC1118. Proc Natl Acad Sci USA 2009, 106(38):16333-16338.

5. Enstero M, Daniel C, Wahlstedt H, Major F, Ohman M: Recognition and coupling of A-to-l edited sites are determined by the tertiary structure of the RNA. Nucleic Acids Res 2009, 37(20):6916-6926.

6. Sugarbaker DJ, Richards WG, Gordon GJ, Dong L, De Rienzo A, Maulik G, Glickman JN, Chirieac LR, Hartman ML, Taillon BE, Du L, Bouffard P, Kingsmore SF, Miller NA, Farmer AD, Jensen RV, Gullans SR, Bueno R: Transcriptome sequencing of malignant pleural mesothelioma tumors. Proc Natl Acad Sci USA 2008, 105(9):3521-3526.

7. Toth AL, Varala K, Newman TC, Miguez FE, Hutchison SK, Willoughby DA, Simons JF, Egholm M, Hunt JH, Hudson ME, Robinson GE: Wasp gene expression supports an evolutionary link between maternal behavior and eusociality. Science 2007, 318(5849):441-444.

8. Zhao Q, Caballero OL, Levy S, Stevenson BJ, Iseli C, de Souza SJ, Galante PA, Busam D, Leversha MA, Chadalavada K, Rogers YH, Venter JC, Simpson AJ, Strausberg RL: Transcriptome-guided characterization of genomic rearrangements in a breast cancer cell line. Proc Natl Acad Sci USA 2009, 106(6):1886-1891.

9. Steuernagel B, Taudien S, Gundlach H, Seidel M, Ariyadasa R, Schulte D, Petzold A, Felder M, Graner A, Scholz U, Mayer KF, Platzer M, Stein N: De novo 454 sequencing of barcoded BAC pools for comprehensive gene survey and genome analysis in the complex genome of barley. $B M C$ Genomics 2009, 10:547.

10. Santana Q, Coetzee M, Steenkamp E, Mlonyeni O, Hammond G, Wingfield $M$, Wingfield B: Microsatellite discovery by deep sequencing of enriched genomic libraries. Biotechniques 2009, 46(3):217-223.

11. Kaneda R, Takada S, Yamashita Y, Choi YL, Nonaka-Sarukawa M, Soda M, Misawa Y, Isomura T, Shimada K, Mano H: Genome-wide histone methylation profile for heart failure. Genes Cells 2009, 14(1):69-77.

12. Schaefer M, Pollex T, Hanna K, Lyko F: RNA cytosine methylation analysis by bisulfite sequencing. Nucleic Acids Res 2009, 37(2):e12.

13. Bundock PC, Eliott FG, Ablett G, Benson AD, Casu RE, Aitken KS, Henry RJ: Targeted single nucleotide polymorphism (SNP) discovery in a highly polyploid plant species using 454 sequencing. Plant Biotechnol J 2009, 7(4):347-354.

14. Sanchez CC, Smith TP, Wiedmann RT, Vallejo RL, Salem M, Yao J, Rexroad CE: Single nucleotide polymorphism discovery in rainbow trout by deep sequencing of a reduced representation library. BMC Genomics 2009, 10:559

15. Noonan JP, Coop G, Kudaravalli S, Smith D, Krause J, Alessi J, Chen F, Platt D, Paabo S, Pritchard JK, Rubin EM: Sequencing and analysis of Neanderthal genomic DNA. Science 2006, 314(5802):1113-1118.
16. Taudien S, Galgoczy P, Huse K, Reichwald K, Schilhabel M, Szafranski K, Shimizu A, Asakawa S, Frankish A, Loncarevic IF, Shimizu N, Siddiqui R, Platzer M: Polymorphic segmental duplications at 8p23.1 challenge the determination of individual defensin gene repertoires and the assembly of a contiguous human reference sequence. BMC Genomics 2004, 5(1):92.

17. Groth M, Huse K, Reichwald K, Taudien S, Hampe J, Rosenstiel P, Birkenmeier G, Schreiber S, Platzer M: Method for preparing singlestranded DNA templates for Pyrosequencing using vector ligation and universal biotinylated primers. Anal Biochem 2006, 356(2):194-201.

18. Huse K, Taudien S, Groth M, Rosenstiel P, Szafranski K, Hiller M, Hampe J, Junker K, Schubert J, Schreiber S, Birkenmeier G, Krawczak M, Platzer M Genetic variants of the copy number polymorphic beta-defensin locus are associated with sporadic prostate cancer. Tumour Bio/ 2008, 29(2):83-92

19. Chen Q, Book M, Fang X, Hoeft A, Stuber F: Screening of copy number polymorphisms in human beta-defensin genes using modified realtime quantitative PCR. J Immunol Methods 2006, 308(1-2):231-240.

20. Fellermann K, Stange DE, Schaeffeler E, Schmalzl H, Wehkamp J, Bevins CL, Reinisch W, Teml A, Schwab M, Lichter P, Radlwimmer B, Stange EF: A chromosome 8 gene-cluster polymorphism with low human betadefensin 2 gene copy number predisposes to Crohn disease of the colon. Am J Hum Genet 2006, 79(3):439-448.

21. Ganz T: Defensins: antimicrobial peptides of innate immunity. Nat Rev Immunol 2003, 3(9):710-720

22. Hollox EJ: Copy number variation of beta-defensins and relevance to disease. Cytogenet Genome Res 2008, 123(1-4):148-155.

23. Hollox EJ, Armour JA, Barber JC: Extensive normal copy number variation of a beta-defensin antimicrobial-gene cluster. Am J Hum Genet 2003, 73(3):591-600.

24. Hollox EJ, Davies J, Griesenbach U, Burgess J, Alton EW, Armour JA: Betadefensin genomic copy number is not a modifier locus for cystic fibrosis. J Negat Results Biomed 2005, 4:9.

25. Hollox EJ, Barber JC, Brookes AJ, Armour JA: Defensins and the dynamic genome: what we can learn from structural variation at human chromosome band 8p23.1. Genome Res 2008, 18(11):1686-1697.

26. Linzmeier RM, Ganz T: Human defensin gene copy number polymorphisms: comprehensive analysis of independent variation in alpha- and beta-defensin regions at 8p22-p23. Genomics 2005, 86(4):423-430.

27. Abu Bakar S, Hollox EJ, Armour JA: Allelic recombination between distinct genomic locations generates copy number diversity in human beta-defensins. Proc Natl Acad Sci USA 2009, 106(3):853-858.

28. Groth M, Szafranski K, Taudien S, Huse K, Mueller O, Rosenstiel P, Nygren AO, Schreiber S, Birkenmeier G, Platzer M: High-resolution mapping of the $8 \mathrm{p} 23.1$ beta-defensin cluster reveals strictly concordant copy number variation of all genes. Hum Mutat 2008, 29(10):1247-1254

29. Hollox EJ, Huffmeier U, Zeeuwen PL, Palla R, Lascorz J, Rodijk-Olthuis D, Kerkhof PC van de, Traupe H, de Jongh G, den Heijer M, Reis A, Armour JA, Schalkwijk J: Psoriasis is associated with increased beta-defensin genomic copy number. Nat Genet 2008, 40(1):23-25.

30. Bentley RW, Pearson J, Gearry RB, Barclay ML, McKinney C, Merriman TR, Roberts RL: Association of higher DEFB4 genomic copy number with Crohn's disease. Am J Gastroenterol 2010, 105(2):354-359.

31. Fredman D, White SJ, Potter S, Eichler EE, Den Dunnen JT, Brookes AJ: Complex SNP-related sequence variation in segmental genome duplications. Nat Genet 2004, 36(8):861-866.

32. Lahr DJ, Katz LA: Reducing the impact of PCR-mediated recombination in molecular evolution and environmental studies using a newgeneration high-fidelity DNA polymerase. Biotechniques 2009 47(4):857-866

33. Schouten JP, McElgunn CJ, Waaijer R, Zwijnenburg D, Diepvens F, Pals G Relative quantification of 40 nucleic acid sequences by multiplex ligation-dependent probe amplification. Nucleic Acids Res 2002, 30(12):e57.

34. Fellermann K, Wehkamp J, Herrlinger KR, Stange EF: Crohn's disease: a defensin deficiency syndrome? Eur J Gastroenterol Hepatol 2003, 15(6):627-634

35. Perne A, Zhang X, Lehmann L, Groth M, Stuber F, Book M: Comparison of multiplex ligation-dependent probe amplification and real-time PCR accuracy for gene copy number quantification using the beta-defensin locus. Biotechniques 2009, 47(6):1023-1028. 
36. Armour JA, Palla R, Zeeuwen PL, den Heijer M, Schalkwijk J, Hollox EJ: Accurate, high-throughput typing of copy number variation using paralogue ratios from dispersed repeats. Nucleic Acids Res 2007, 35(3):e19.

37. Goossens D, Moens LN, Nelis E, Lenaerts AS, Glassee W, Kalbe A, Frey B, Kopal G, De Jonghe P, De Rijk P, Del-Favero J: Simultaneous mutation and copy number variation (CNV) detection by multiplex PCR-based GS-FLX sequencing. Hum Mutat 2009, 30(3):472-476.

38. Thomas RK, Nickerson E, Simons JF, Janne PA, Tengs T, Yuza Y, Garraway LA, LaFramboise T, Lee JC, Shah K, O'Neill K, Sasaki H, Lindeman N, Wong KK, Borras AM, Gutmann EJ, Dragnev KH, DeBiasi R, Chen TH, Glatt KA, Greulich H, Desany B, Lubeski CK, Brockman W, Alvarez P, Hutchison SK, Leamon JH, Ronan MT, Turenchalk GS, Egholm M, et al: Sensitive mutation detection in heterogeneous cancer specimens by massively parallel picoliter reactor sequencing. Nat Med 2006, 12(7):852-855

39. Binladen J, Gilbert MT, Bollback JP, Panitz F, Bendixen C, Nielsen R, Willerslev E: The use of coded PCR primers enables high-throughput sequencing of multiple homolog amplification products by 454 parallel sequencing. PLoS One 2007, 2(2):e197.

40. International_HapMap_Project: The International HapMap Project. Nature 2003, 426(6968):789-796

41. European Collection of Cell Cultures (ECACC) [http:// www.hpacultures.org.uk/collections/ecacc.jsp]

42. Aldhous MC, Noble CL, Satsangi J: Dysregulation of human betadefensin-2 protein in inflammatory bowel disease. PLoS One 2009, 4(7):e6285.

43. Wang G, Stange EF, Wehkamp J: Host-microbe interaction: mechanisms of defensin deficiency in Crohn's disease. Expert Rev Anti Infect Ther 2007, 5(6):1049-1057.

44. Wehkamp J, Koslowski M, Wang G, Stange EF: Barrier dysfunction due to distinct defensin deficiencies in small intestinal and colonic Crohn's disease. Mucosal Immunol 2008, 1(Suppl 1):S67-74.

45. Horton R, Gibson R, Coggill P, Miretti M, Allcock RJ, Almeida J, Forbes S, Gilbert JG, Halls K, Harrow JL, Hart E, Howe K, Jackson DK, Palmer S, Roberts AN, Sims S, Stewart CA, Traherne JA, Trevanion S, Wilming L, Rogers J, de Jong PJ, Elliott JF, Sawcer S, Todd JA, Trowsdale J, Beck S: Variation analysis and gene annotation of eight MHC haplotypes: the MHC Haplotype Project. Immunogenetics 2008, 60(1):1-18.

46. Barnard R, Futo V, Pecheniuk N, Slattery M, Walsh T: PCR bias toward the wild-type k-ras and $\mathrm{p} 53$ sequences: implications for PCR detection of mutations and cancer diagnosis. Biotechniques 1998, 25(4):684-691.

47. Warnecke PM, Stirzaker C, Melki JR, Millar DS, Paul CL, Clark SJ: Detection and measurement of PCR bias in quantitative methylation analysis of bisulphite-treated DNA. Nucleic Acids Res 1997, 25(21):4422-4426.

48. Alkan C, Kidd JM, Marques-Bonet T, Aksay G, Antonacci F, Hormozdiari F, Kitzman JO, Baker C, Malig M, Mutlu O, Sahinalp SC, Gibbs RA, Eichler EE: Personalized copy number and segmental duplication maps using next-generation sequencing. Nat Genet 2009, 41(10):1061-1067.

doi: 10.1186/1471-2164-11-252

Cite this article as: Taudien et al., Haplotyping and copy number estimation of the highly polymorphic human beta-defensin locus on 8 p23 by 454 amplicon sequencing BMC Genomics 2010, 11:252

Submit your next manuscript to BioMed Central and take full advantage of:

- Convenient online submission

- Thorough peer review

- No space constraints or color figure charges

- Immediate publication on acceptance

- Inclusion in PubMed, CAS, Scopus and Google Scholar

- Research which is freely available for redistribution

Submit your manuscript at www.biomedcentral.com/submit
C Biomed Central 